\title{
Expression of indoleamine 2,3-dioxygenase by plasmacytoid dendritic cells in tumor-draining lymph nodes
}

\author{
David H. Munn, 1,2,3 Madhav D. Sharma, 1,2 Deyan Hou,1,2 Babak Baban, ${ }^{2}$ \\ Jeffrey R. Lee,2,3,4 Scott J. Antonia, ${ }^{5}$ Jane L. Messina, ${ }^{5}$ Phillip Chandler, ${ }^{2,6}$ \\ Pandelakis A. Koni, ${ }^{2,6}$ and Andrew L. Mellor ${ }^{2,6}$

\begin{abstract}
'Department of Pediatrics, ${ }^{2}$ Institute for Molecular Medicine and Genetics, and ${ }^{3}$ Department of Pathology, Medical College of Georgia, Augusta, Georgia, USA. ${ }^{4}$ Augusta Veterans Affairs Medical Center, Augusta, Georgia, USA. ${ }^{5}$ H. Lee Moffitt Cancer Center, Tampa, Florida, USA.
\end{abstract} \\ ${ }^{6}$ Department of Medicine, Medical College of Georgia, Augusta, Georgia, USA.
}

\begin{abstract}
One mechanism contributing to immunologic unresponsiveness toward tumors may be presentation of tumor antigens by tolerogenic host APCs. We show that mouse tumor-draining LNs (TDLNs) contained a subset of plasmacytoid DCs (pDCs) that constitutively expressed immunosuppressive levels of the enzyme indoleamine 2,3-dioxygenase (IDO). Despite comprising only $0.5 \%$ of $L N$ cells, these pDCs in vitro potently suppressed $\mathrm{T}$ cell responses to antigens presented by the pDCs themselves and also, in a dominant fashion, suppressed $\mathrm{T}$ cell responses to third-party antigens presented by nonsuppressive APCs. Adoptive transfer of DCs from TDLNs into naive hosts created profound local $T$ cell anergy, specifically toward antigens expressed by the transferred DCs. Anergy was prevented by targeted disruption of the IDO gene in the DCs or by administration of the IDO inhibitor drug 1-methyl-D-tryptophan to recipient mice. Within the population of pDCs, the majority of the functional IDO-mediated suppressor activity segregated with a novel subset of pDCs coexpressing the B-lineage marker CD19. We hypothesize that IDO-mediated suppression by pDCs in TDLNs creates a local microenvironment that is potently suppressive of host antitumor $\mathrm{T}$ cell responses.
\end{abstract}

\section{Introduction}

The immune system of tumor-bearing hosts fails to respond protectively to tumor antigens. Functionally, the host is tolerant toward the tumor (1). This is not due to a peculiarity of tumor antigens, since even highly immunogenic viral proteins can become tolerizing when introduced on tumors (2). The molecular mechanisms underlying tumor-induced tolerance are still poorly understood. Contributing factors may include a component of immunologic "ignorance" (3-5), particularly in the early phase of tumor growth, as well as active suppression by regulatory T cells (Treg's) (6). In addition, it has been proposed that certain host APCs may present tumor antigens in a fashion that leads to tolerance (7-9).

Presentation of tumor antigens by host APCs (indirect presentation, or cross-presentation) is a key step by which naive T cells first become aware of tumor-derived antigens. Cross-presentation can lead to activation of tumor-specific T cells $(10,11)$, although usually without regression of established tumors, or it can lead to anergy $(8,9)$. DCs are the key APCs for naive T cells $(12,13)$, and it is now clear that DCs may be either activating or tolerizing (14). However, the specific role of tolerogenic DCs in tumor immunology is not yet well defined.

In part, this may be because the mechanisms by which DCs create tolerance in T cells are still poorly understood (15). In the current study we focus on the tryptophan-degrading enzyme indoleamine

Nonstandard abbreviations used: indoleamine 2,3-dioxygenase (IDO); 1-methyl-D-tryptophan (1MT); mixed leukocyte reaction (MLR); plasmacytoid DC (pDC); regulatory T cell (Treg); T cell receptor (TCR); tumor-draining LN (TDLN). Conflict of interest: The authors have declared that no conflict of interest exists. 2,3-dioxygenase (IDO), an immunosuppressive mechanism shared by several different cell types in the immune system $(16,17)$. Expression of IDO by certain types of human monocyte-derived macrophages and in vitro-derived DCs allows them to inhibit $\mathrm{T}$ cell proliferation (18-20). In vivo, endogenous IDO has been implicated in maternal tolerance toward the allogeneic fetus (21); in tolerance to self antigens in NOD mice (22); as a downstream effector mechanism for the tolerance-inducing agent CTLA4-Ig (23); and as a protective negative regulator of experimentally induced autoimmune disorders $(24,25)$.

Based on this, we and others have hypothesized that IDO may be exploited by tumors as a mechanism of immune evasion (26). Consistent with this possibility, IDO has been shown to be expressed by a variety of primary human tumors and human tumor cell lines $(27,28)$. Transfection of recombinant IDO into murine tumor cell lines confers the ability to inhibit antigen-specific $\mathrm{T}$ cell responses in vitro (29). In vivo, IDO-transfected tumor cells are able to escape immune rejection, even in previously immunized hosts that would normally be fully protected against tumor challenge (27). These data suggest that expression of IDO by malignant cells could contribute to local immunosuppression at the site of tumors.

However, expression of IDO by the tumor cells themselves would not affect cross-presentation by host APCs. To influence this critical regulatory step, IDO would need to be expressed in host DCs in tumor-draining LNs (TDLNs). Consistent with this possibility, we have reported that certain patients display a prominent population of IDO-expressing host-derived mononuclear cells in TDLNs $(20,30)$. Similar cells are also seen in murine TDLNs (31). However, the immunologic role of these cells is unknown. In the current study we use a murine tumor model to test the hypothesis that IDO-expressing cells in TDLNs represent a population of tolerogenic host DCs. 
A

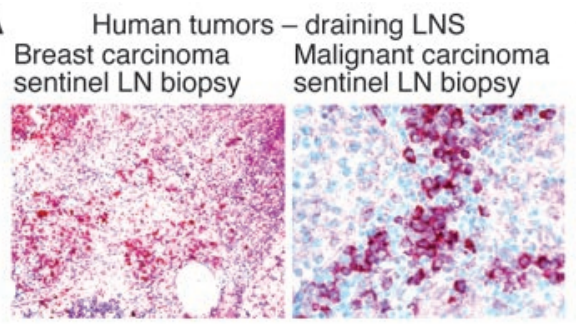

B

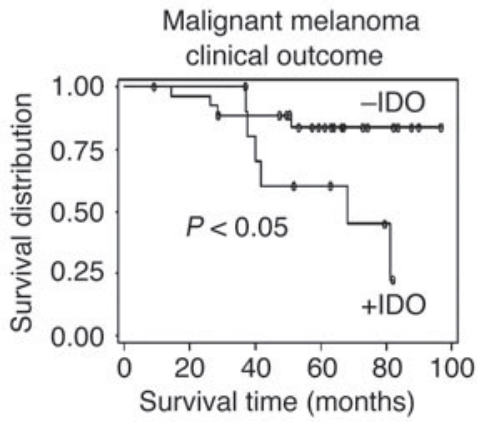

C
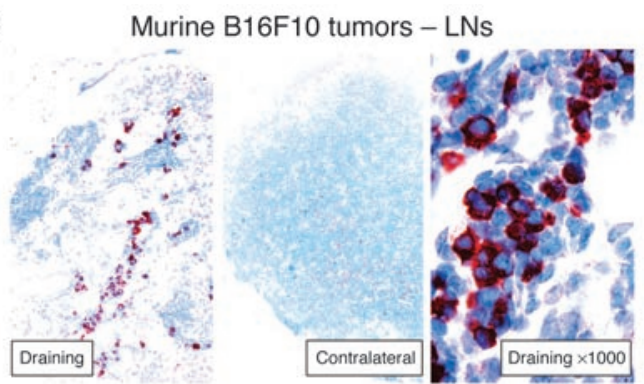

D

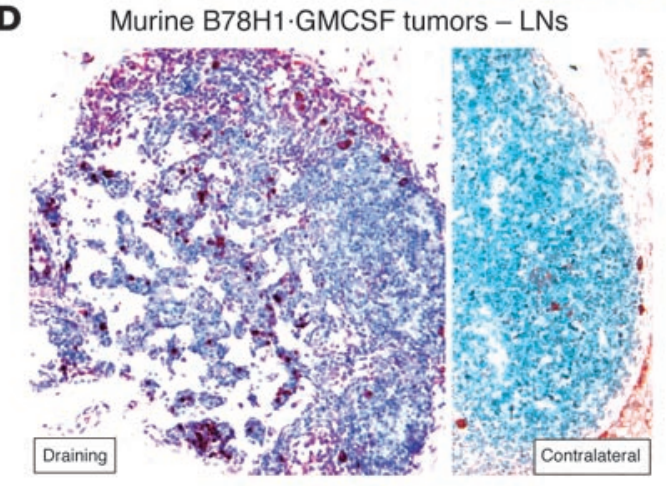

\section{Figure 1}

Expression of IDO in human and murine TDLNs. (A) Sentinel (first draining) LN from patients with breast carcinoma (left, $\times 100$ ) and malignant melanoma (right, $\times 400$ ), showing an abnormal infiltration of IDO ${ }^{+}$cells (red chromogen). (B) Kaplan-Meier survival plot of 40 patients with malignant melanoma, stratified into those with an abnormal accumulation of IDO+ cells in the sentinel LN (+IDO), versus a normal (negative) pattern. (C) Expression of IDO in murine B16F10 melanoma. Left: Draining inguinal LN from a mouse with a B16F10 tumor, day 12, stained for IDO (red, $\times 100$ ). Middle: Contralateral inguinal LN from the same animal as at left, stained for IDO (red, $\times 100)$. Right: High-power view of IDO+ cells shown in the left panel $(\times 1,000)$. Controls for staining (anti-IDO antibody neutralized with the immunizing peptide) showed a negative pattern similar to that seen in the contralateral LN (not shown). (D) Draining and contralateral LNs from a mouse with B78H1.GM-CSF tumor, day 12, stained for IDO (red, both ×200).

\section{Results}

IDO-expressing cells in human TDLNs correlate with a worse clinical outcome. The starting point for our murine studies was the clinical observation that a subset of patients with malignant tumors (breast carcinoma, malignant melanoma, and a number of other solid tumors) displayed abnormal accumulation of IDO-expressing cells in the LNs draining the tumors (Figure 1A). A pilot study was performed using archival specimens from 40 patients with malignant melanoma. Figure $1 \mathrm{~B}$ shows that the presence of abnormal IDO ${ }^{+}$cells in the sentinel LN at the time of initial diagnosis correlated with a significantly worse long-term outcome. None of these patients had detectable LN metastases at the time of diagnosis (by H\&E and immunohistochemistry), which suggested that the recruitment of $\mathrm{IDO}^{+}$cells occurred early in the course of the disease. While this small pilot study was not intended to validate IDO as a clinical prognostic marker, the findings were consistent with the possibility that IDO might play a mechanistic role in the biology of certain tumors. This prompted the development of a defined mouse model in which to test this hypothesis.

IDO-expressing cells in murine TDLNs. Figure $1 \mathrm{C}$ shows that $\mathrm{IDO}^{+}$cells were found in TDLNs of established B16F10 mouse melanomas. The contralateral (non-tumor-draining) LNs from the same animals showed few or no IDO-expressing cells (Figure 1C). Although the $\mathrm{IDO}^{+}$cells were morphologically similar to the plasmacytoid-appearing cells seen in humans, the absolute number of these cells found in the B16F10 model was much lower than in heavily infiltrated human tumor-bearing C57BL/6 mice, and preliminary validation studies confirmed that expression of $\mathrm{H} 2 \mathrm{~K}^{\mathrm{b}}$ was identical in DCs from TDLNs and normal LNs.) As shown in Figure 2A, TDLN cells appeared to be poor stimulators of BM3 T cells, whereas cells from the contralateral LNs of the same animals were excellent stimulators (comparable to LN cells from non-tumor-bearing mice, not shown).

The lack of response to TDLN cells might be due either to inadequate APC function, or to some form of active suppression. Mixing experiments (Figure 2A, right panel) showed that the lack of proliferation was due to a dominant suppressor activity present in the TDLN cells. Sorting experiments (Figure $2 \mathrm{~B}$ ) revealed that one component of this suppression segregated with the $\mathrm{CD} 25^{+} \mathrm{CD} 4^{+}$(Treg) fraction (as expected, since Treg's are known to be present in mice with B16 tumors [ref. 35]). However, removing the Treg's did not abrogate the suppressor activity. Further fractionation revealed the existence of a second, equally potent suppressor activity associated with the $\mathrm{CD} 11 \mathrm{c}^{+} \mathrm{B} 220^{+}$fraction, which is the phenotype of murine plasmacytoid DCs ( $\mathrm{pDCs})$. This population contained all of the remaining suppressor activity, such that when both the $\mathrm{pDCs}$ and the Treg's were removed, the remaining 95-97\% of TDLN cells (which included all the APCs other than pDCs) stimulated excellent proliferation. Mixing experiments showed that suppression mediated by pDCs was dominant over stimulation mediated by all other APCs: i.e., admixture of the $<1 \%$ fraction of $\mathrm{pDCs}$ with the $>95 \%$ of other, nonsuppressive cells inhibited $\mathrm{T}$ cell responses to both populations (Figure 2B). 
A

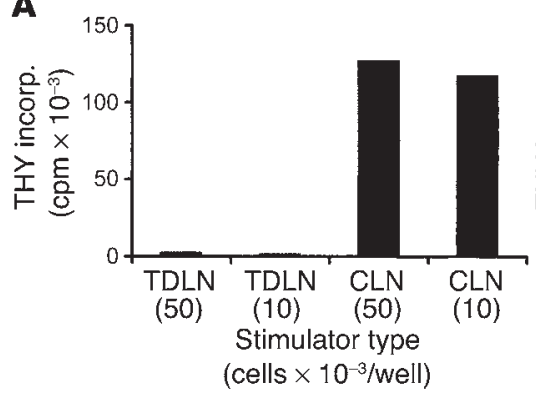

B

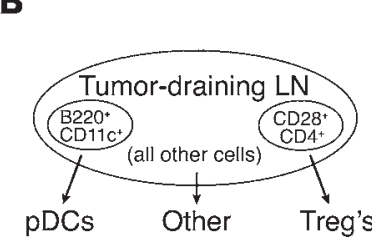

$$
\text { 156 }
$$

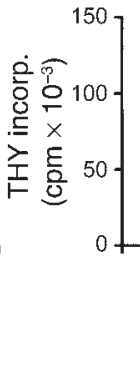

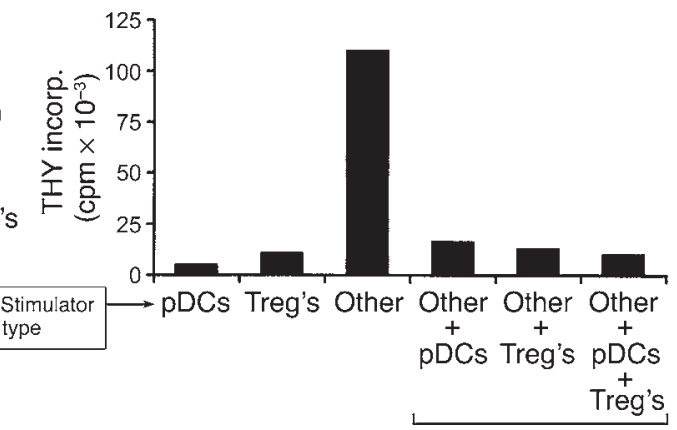

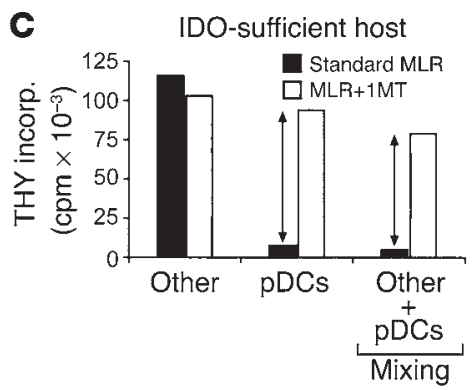

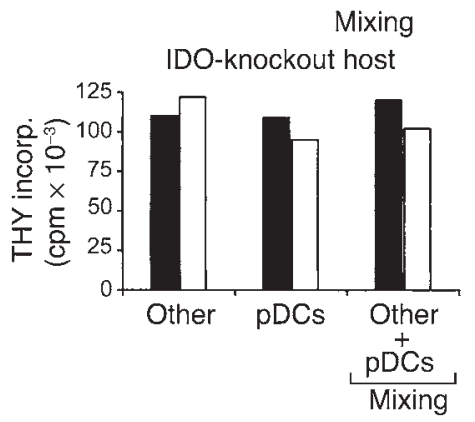

Figure 2

Suppression of T cell responses by TDLN cells. (A) Cells from TDLNs and contralateral LNs (CLN) were harvested from mice with B78H1.GM-CSF tumors (day 14), and used as stimulators in MLRs (stimulator cell number in parentheses; responder BM3 T cells at $5 \times 10^{4}$ per well). Mixing experiments (right) demonstrated dominant suppression by the TDLN cells. (B) TDLN cells were sorted by four-color flow cytometry into pDCs $(<1 \%)$, Treg's (2-3\%), and all other cells (95-97\%), using the markers shown. Each fraction was used as stimulator cells in MLRs, adding the number of cells that would have been present in $5 \times 10^{4}$ of the original TDLN population (thus, the pDCs were added at approximately 500 cells per well). All MLRs received $5 \times 10^{4}$ BM3 responders. For mixing experiments, the sorted fractions were mixed in the same ratio in which they were present in the original unsorted preparation. (C) Suppression by pDCs is mediated by IDO. TDLN cells were sorted as described for $\mathbf{B}$. The pDCs and "other" fraction were used as stimulators in MLRs (BM3 responders), with and without the IDO inhibitor 1MT. Left: TDLN cells taken from a wild-type (IDO-sufficient) host, showing suppression by pDCs, which was blocked by $1 \mathrm{MT}$ (arrows), and dominant suppression with mixing. Right: Tumors were grown in IDO-KO hosts, and the IDO-KO TDLN cells were harvested and assayed as at left. THY incorp., thymidine incorporation.
Immunosuppression by pDCs is mediated by IDO. To test the role of IDO in this system, MLRs were performed in the presence or absence of the IDO inhibitor 1-methyl-D-trytophan (1MT). Figure 2C shows that $1 \mathrm{MT}$ blocked the inhibitory activity of $\mathrm{pDCs}$, converting them into potent stimulators of $\mathrm{T}$ cell proliferation. When $\mathrm{pDCs}$ and the nonsuppressive "other" fraction were mixed together, 1MT reversed the dominant suppression mediated by pDCs. The effect of $1 \mathrm{MT}$ was not due to nonspecific activation of T cells, since the same T cells stimulated by the nonsuppressive "other" fraction showed no effect of 1MT (Figure 2C). Next, tumors were implanted in mice with a targeted disruption of the IDO gene (IDO-KO mice). The pDCs isolated from TDLNs of IDO-KO mice showed no suppressor activity (Figure $2 \mathrm{C}$, right panel), confirming that suppression required an intact IDO gene. Likewise, $1 \mathrm{MT}$ had no effect on MLRs stimulated by the IDO-KO DCs, confirming the specificity of 1MT for IDO.

In other experiments (not shown in the interest of space), sorted pDCs from TDLNs pulsed with SIINFEKL peptide inhibited proliferation of OVA-specific CD8 ${ }^{+}$OT-I T cells (36) by more than $90 \%$ compared with nonsuppressive APC controls. Just as with BM3 T cells, inhibition was fully reversed by $1 \mathrm{MT}$, there was no inhibition by the B220- (nonplasmacytoid) fraction of DCs from the same TDLNs, and there was no inhibition by B220 ${ }^{+}$pDCs when they were derived from normal LNs (C57BL/6 mice without tumors).

IDO can suppress third-party T cells. The mixing experiments in Figure $2 \mathrm{C}$ suggested that IDO could inhibit $\mathrm{T}$ cell responses even to antigens presented by neighboring, nonsuppressive APCs. To test this hypothesis, we performed mixing experiments using a defined population of third-party $T$ cells that could only see antigen presented by nonsuppressive (IDO-) APCs. IDO ${ }^{+} \mathrm{pDCs}$ and responder BM3 cells, as in the preceding experiments, were mixed with thirdparty TCR-transgenic $\mathrm{CD}^{+}{ }^{+} \mathrm{T}$ cells (CBA background) recognizing an HY-derived peptide presented by $\mathrm{H} 2 \mathrm{E}^{\mathrm{k}}$. APCs for these cells were nonsuppressive CD $11 \mathrm{c}^{+} \mathrm{DCs}$ from normal CBA spleen. (This experiment is shown schematically in Figure 3.) When the two sets of APC/responder pairs were mixed together, the IDO-expressing pDCs dominantly suppressed the HY-specific T cell proliferation, in a progressive dose-dependent fashion (Figure 3). Suppression was mediated by IDO, as shown by its reversal with $1 \mathrm{MT}$.

Adoptive transfer of DCs from TDLNs creates immunologic unresponsiveness in vivo. We next asked whether the DCs isolated from TDLNs were able to create immunologic unresponsiveness in new hosts without tumors. CD $11 \mathrm{c}^{+}$DCs were isolated from TDLNs of $\mathrm{H} 2 \mathrm{~K}^{\mathrm{b}}$ positive hosts and adoptively transferred to naive, allogeneic CBA hosts $\left(\mathrm{H} 2 \mathrm{~K}^{\mathrm{b}}\right.$-negative). Recipients were preloaded with a cohort of $\mathrm{H} 2 \mathrm{~K}^{\mathrm{b}}$-specific BM3 $\mathrm{T}$ cells, so that defined antigen-specific responses could be followed. We chose an alloantigen for these studies (rather than a cross-presented tumor antigen) because the alloantigen was constitutively expressed on all DCs, thus ensuring that it would not be lost after adoptive transfer. To test whether the $\mathrm{IDO}^{+}$DCs would dominantly suppress all antigen-specific T cell 
responses (even to $\mathrm{H} 2 \mathrm{~K}^{\mathrm{b}}$ presented by nonsuppressive APCs in the transferred population, as predicted by Figure 3), we transferred all of the CD $11 \mathrm{c}^{+} \mathrm{DC}$ from TDLNs, not just the $\mathrm{IDO}^{+} \mathrm{pDC}$ fraction.

Ten days after adoptive transfer, $T$ cells were isolated from recipients. Figure 4A shows that the transferred DCs induced selective accumulation of antigen-specific BM3 T cells in the LNs draining the site of injection (inguinal LNs). This accumulation was comparable whether the transferred DCs were derived from TDLNs or from normal C57BL/6 LNs, but it did not occur when DCs were derived from antigen-negative CBA mice. In all groups, the number of BM3 T cells in host spleens was similar, indicating that the initial systemic loading of BM3 was comparable.

Inguinal LN cells from recipient mice were assayed for responsiveness in MLRs against $\mathrm{H} 2 \mathrm{~K}^{\mathrm{b}}$-positive targets (irradiated C57BL/ 6 splenocytes). Figure 4B shows that $\mathrm{T}$ cells from mice receiving TDLN DCs displayed profound hyporesponsiveness to recall antigen, despite the fact that there were ample BM3 $\mathrm{T}$ cells present in the LNs (Figure 4A). In contrast, T cells from LNs of animals receiving normal C57BL/6 DCs displayed a brisk MLR response. Mice receiving antigen-negative DCs also responded well in MLRs, showing that the large cohort of pre-positioned BM3 cells allowed a vigorous ex vivo response, without the need for previous priming. Thus, the lack of response in the mice receiving TDLN DCs represented the active elimination of a preexisting state of responsiveness, not a failure of priming.

Creation of immunologic unresponsiveness is IDO-dependent. To test whether this acquired unresponsiveness was caused by IDO, recipient mice were treated with the IDO inhibitor $1 \mathrm{MT}$, beginning at the time of adoptive transfer. After 10 days, $\mathrm{T}$ cells were harvested and tested for response to antigen. Figure 4C shows that administration of $1 \mathrm{MT}$ in vivo prevented the induction of $\mathrm{T}$ cell unresponsiveness by TDLN DCs. In contrast, $1 \mathrm{MT}$ had no effect on mice receiving normal DCs (right panels). In all experiments, flow cytometry confirmed that LNs from all groups contained comparable numbers of BM3 T cells (not shown). In the same experiments, we tested the response of systemic $\mathrm{T}$ cells from a remote site (spleen). Figure 4C (lower panels) shows that splenic T cells from animals primed with TDLN DCs displayed modestly reduced responses compared with controls, and that this was prevented by 1MT administration; however, splenic T cells did not show the complete unresponsiveness of cells from draining LNs.

The profound unresponsiveness created in draining LNs following adoptive transfer was prevented by coadministration of $1 \mathrm{MT}$ (and was thus IDO-dependent), but it was not clear whether the relevant IDO was expressed by the adoptively transferred DCs, or by some host cell. To test this, B78H1.GM-CSF tumors were grown in IDO-KO mice, and then the IDO-KO TDLN DCs were harvested and transferred into $\mathrm{CBA}+\mathrm{BM} 3$ hosts, exactly as in the preceding experiments. Figure 4D shows that IDO-deficient DCs from TDLNs did not create unresponsiveness in the recipient $\mathrm{T}$ cells, despite the fact that the tumor, the CBA recipients, and the BM3 T cells were all wild-type for IDO. Thus, the relevant site of expression for IDO was in the transferred DCs. Further, the only mechanistic intervention required to render TDLN DCs nonsuppressive was the removal of IDO (either by knockout or by $1 \mathrm{MT}$ ).

$I D \mathrm{O}^{+} \mathrm{DCs}$ create antigen-specific anergy in draining $L N s$. The profound $\mathrm{T}$ cell unresponsiveness created following adoptive transfer was not due simply to carry-over of the original $\mathrm{IDO}^{+} \mathrm{DCs}$, as shown by the fact that $\mathrm{T}$ cell proliferation was not restored by addition of $1 \mathrm{MT}$ to the recall MLRs (Figure 5A). (This was in con- trast to the effect of $1 \mathrm{MT}$ administered in vivo, during the afferent priming phase, where IDO was absolutely required; compare Figure 4C.) To test whether this unresponsiveness reflected antigen-specific anergy, LN cells were harvested 10 days after priming with TDLN DCs, and the BM3 T cells were sorted to greater than $95 \%$ purity based on expression of clonotypic TCR. Figure $5 \mathrm{~B}$ shows that purified BM3 $\mathrm{T}$ cells were anergic to stimulation by irradiated C57BL/ 6 spleen cells and were also refractory to mitogenic anti-CD3 antibody, or to stimulation with PMA plus ionomycin (which bypasses the need for TCR signaling). In all of these models, however, proliferation was restored by the addition of recombinant IL-2 to the MLRs. The unresponsive state of BM3 $\mathrm{T}$ cells was thus consistent with classical descriptions of clonal anergy (37) and was also consistent with tumor-induced anergy observed in other systems (38).

To test whether anergy was created in an antigen-specific fashion, we asked whether the other host $\mathrm{CD}^{+} \mathrm{T}$ cells in the same LNs retained responsiveness. (For these experiments we used the unfractionated $\mathrm{CD}^{+}$cells from the same LNs as the anergic BM3 cells, which would include all the host $\mathrm{CD}^{+} \mathrm{T}$ cells that had not recognized antigen on the transferred DCs.) Figure 5C shows that the unfractionated host $\mathrm{CD}^{+} \mathrm{T}$ cells remained fully responsive to mitogenic anti-CD3 antibody, despite anergy of the purified BM3 cells from the same node. Likewise, the response of LN cells to irrelevant $(\mathrm{BALB} / \mathrm{c})$ antigens was comparable between hosts receiving TDLN DCs and normal, untreated CBA mice (Figure 5D).

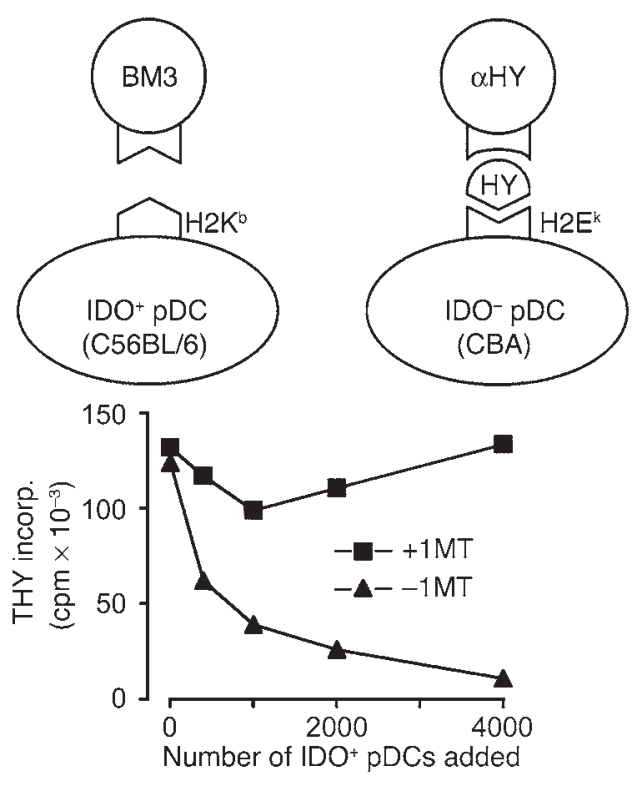

Figure 3

Dominant third-party suppression mediated by IDO+ ${ }^{+}$DCs. Two independent pairs of APCs and responder T cells were used to test the effect of IDO on third-party $T$ cell responses. IDO+ ${ }^{+}$DCs from TDLNs presented $\mathrm{H}_{2} \mathrm{~K}^{\mathrm{b}}$ antigen to BM3 T cells, while IDO- DCs (sorted CD11 $\mathrm{C}^{+}$cells from normal CBA spleen) presented antigen to TCRtransgenic $\mathrm{CD}_{4}{ }^{+} \mathrm{T}$ cells (recognizing a peptide from $\mathrm{HY}$, restricted on $\mathrm{H}_{2} \mathrm{E}^{\mathrm{k}}$ ). In both cases, the APC/T cell ratio was 1:25. Graded numbers of the $\mathrm{pDC}+\mathrm{BM} 3$ pair were added to $1 \times 10^{5}$ cells of the $\mathrm{DC}+\alpha \mathrm{HY}$ pair, and the total proliferation was measured after 72 hours. The number of IDO+ pDCs added is shown on the $x$ axis (with 4,000 cells reflecting a $1: 1$ ratio between the two pairs of MLRs). Duplicate sets of wells received either $1 \mathrm{MT}$ (squares) or no $1 \mathrm{MT}$ (triangles). 

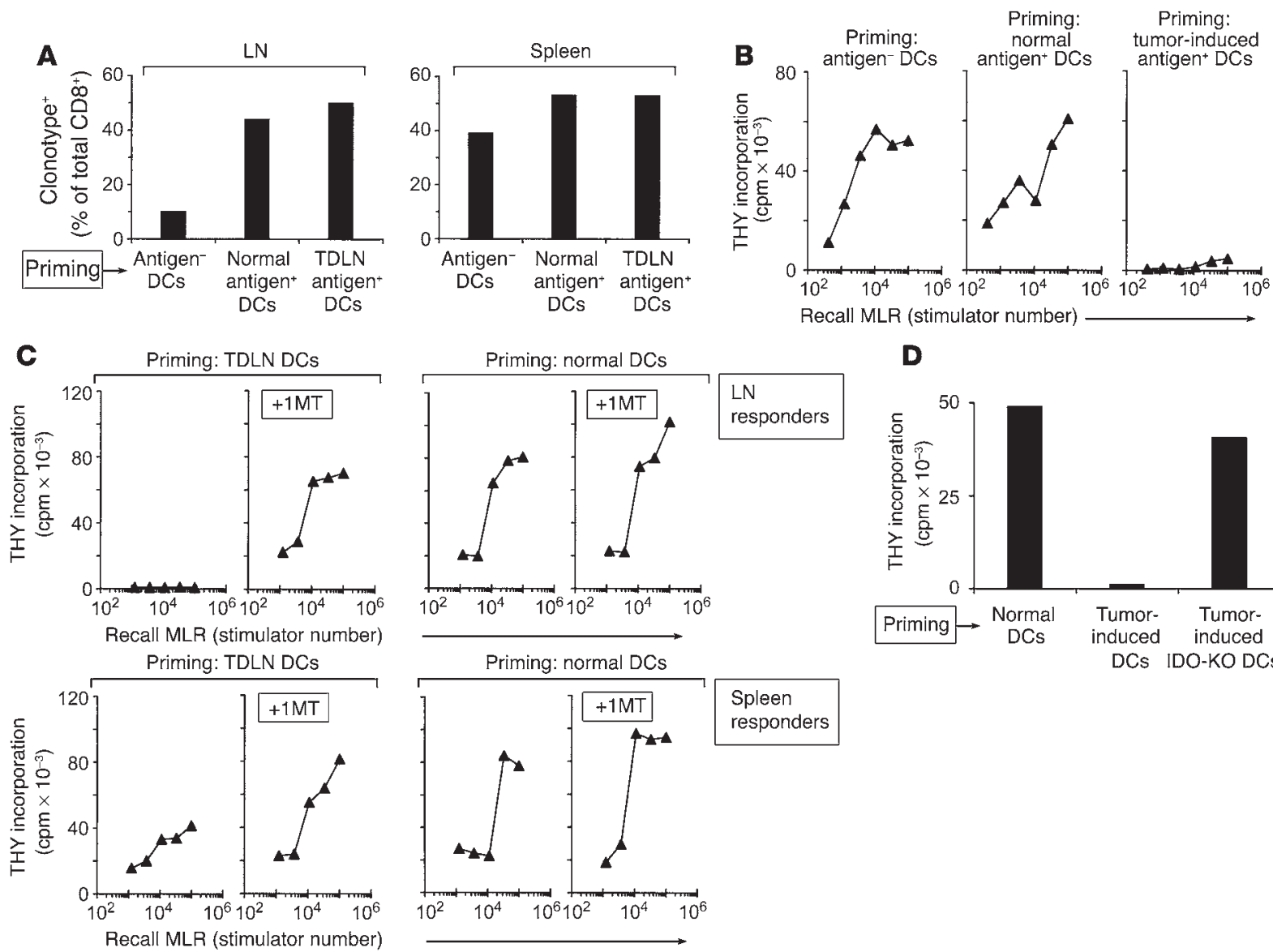

D
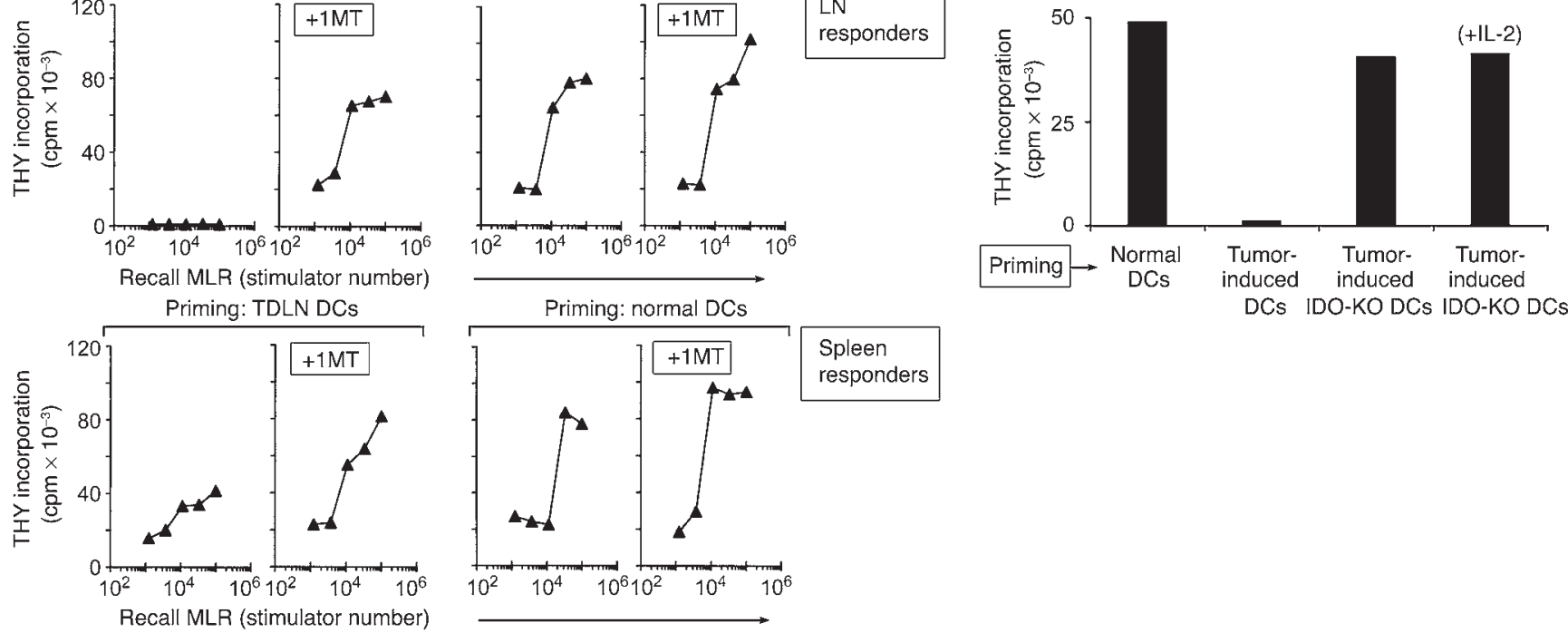

DCs IDO-KO DCs IDO-KO DCs

Figure 4

Adoptive transfer of DCs from TDLNs creates immunosuppression in new hosts. (A) Recruitment of BM3 T cells to draining LNs. CD11c ${ }^{+}$DCs were purified from TDLNs and injected subcutaneously into CBA mice; recipients had previously received $4 \times 10^{7}$ BM3 splenocytes intravenously (CBA+BM3 hosts). Control recipients received normal CD11 ${ }^{+}$DCs from antigen-postive C57BL/6 mice without tumors (Normal antigen+ DCs); or normal DCs from antigen-negative CBA mice (Antigen- DCs). After 10 days, LNs draining the site of DC injection (left) and spleens (right) were harvested. BM3 cells were enumerated by FACS using anti-clonotypic antibody (expressed as a percentage of the total CD8 ${ }^{+} \mathrm{T}$ cells). Each bar represents four pooled nodes. (B) Functional unresponsiveness of T cells primed with DCs from TDLNs. CBA+BM3 mice were primed as described above, and LN cells were used as responders in recall MLRs $\left(1 \times 10^{5}\right.$ responder cells with a titration of irradiated C57BL/6 splenocyte stimulators). (C) CBA+BM3 mice were primed for 10 days with DCs from TDLNs (left) or normal C57BL/6 LNs (right). Half of each group received $1 \mathrm{MT}$ ( $5 \mathrm{mg} / \mathrm{d}$ ) via subcutaneous pellet as described in Methods, from the time of adoptive transfer until the end of the experiment; the other half received vehicle alone. Recall MLRs were performed as above. (D) Creation of unresponsiveness required functional IDO in the transferred DCs. Tumors were grown in IDO-KO mice, and TDLN DCs were isolated and used to prime CBA+BM3 mice, as in the preceding panels. Control recipients received TDLN DCs from wild-type hosts, or normal DCs from non-tumor-bearing mice. Just as above, normal DCs did not create unresponsiveness in recall MLRs, and IDO-sufficient TDLN DCs created complete unresponsiveness. The IDO-KO DCs, even though from TDLNs, did not create unresponsiveness; and responses were not further enhanced by addition of exogenous IL-2 to the recall MLR (last bar), which argues against any component of partial or cryptic anergy.

IDO-mediated suppression preferentially segregates with a subset of $C D 19^{+} D C s$. The experiments shown in Figure 2B suggested that IDO-mediated suppression segregated with the plasmacytoid $\left(\mathrm{B} 220^{+}\right)$DC fraction. To better define the specific cell type responsible for suppression, we performed further phenotyping studies. These revealed that the pDCs from TDLNs were heterogeneous in expression of a number of markers, but in particular CD19. To determine whether this heterogeneity corresponded to functional differences, pDCs from TDLN cells were sorted into the CD19+ and CD19- fractions, plus the conventional B220-CD $11 \mathrm{c}^{+} \mathrm{DCs}$, as depicted schematically in Figure 6. B cells were also isolated, for use as IDO-negative controls. In preliminary studies, both the $\mathrm{CD} 19^{+}$and the $\mathrm{CD} 19^{-}$pDCs were found to express IDO by immunohistochemistry and Western blot, and some of the B220$\mathrm{DCs}$ were $\mathrm{IDO}^{+}$as well. However, it has been shown that IDO protein may be detectable without functional IDO-mediated immunosuppression (39), so it was important to perform functional studies of each subset. When each sorted population was used as APCs in MLRs, virtually all of the functional IDO-mediated suppression was found to segregate with the $\mathrm{CD} 19^{+}$fraction of $\mathrm{pDCs}$. When these cells were removed, the remaining DCs showed little suppression (Figure 6, groups II and III).

Despite thus comprising virtually all of the IDO-mediated suppressor activity, CD $19^{+}$pDCs represented only a small fraction of total LN cells. In 13 experiments, analyzing two to six pooled TDLNs each, the total CD $11 \mathrm{c}^{+} \mathrm{DC}$ fraction was found to constitute 

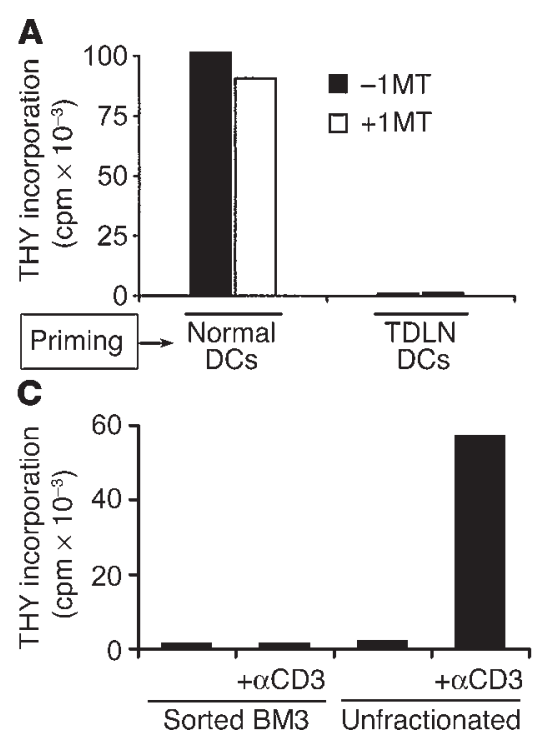
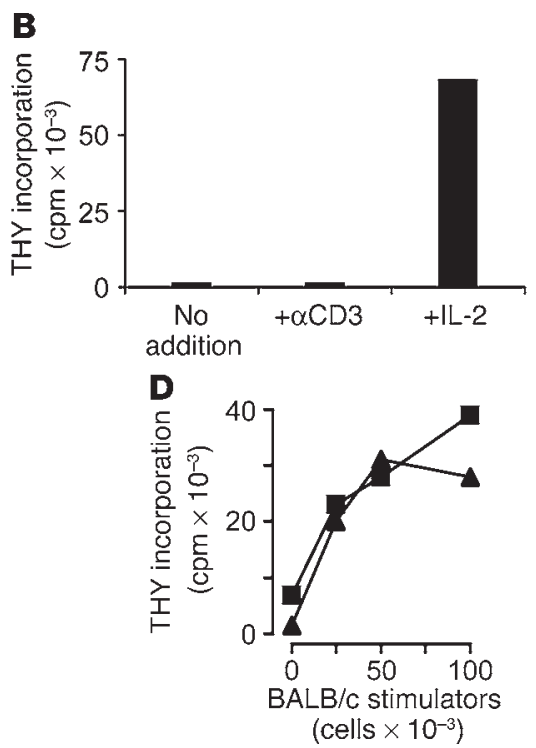
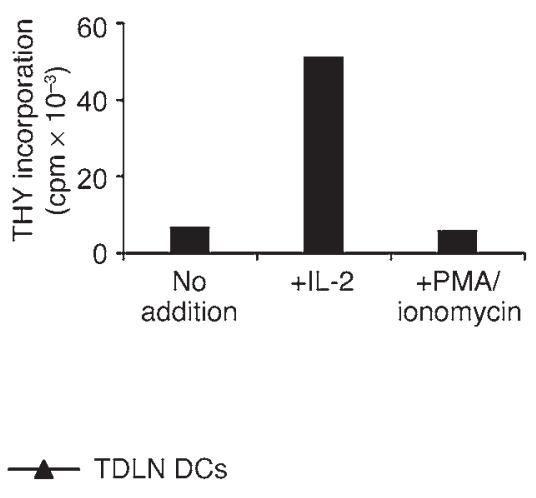

$\rightarrow-$ Normal CBA

Figure 5

Antigen-specific anergy induced by adoptive transfer of TDLN DCs. (A) T cell unresponsiveness following adoptive transfer was not due to carry-over of IDO-mediated suppression, as shown by the lack of effect of $1 \mathrm{MT}$ when added to the recall MLRs. Priming of CBA+BM3 recipients was with TDLN DCs or normal C57BL/6 DCs, as in the previous figures. (B) Anergic BM3 cells are rescued by exogenous IL-2. CBA+BM3 recipients were primed with TDLN DCs, and BM3 T cells were sorted from draining LNs based on clonotypic TCR expression versus CD8. Recall MLRs were performed using these purified BM3 responders and irradiated C57BL/6 spleen cell stimulators, with or without the addition of mitogenic anti-CD3 antibody, recombinant IL-2, or PMA/ionomycin to the MLRs, as shown. (C) CBA+BM3 hosts were primed with TDLN DCs, and then anergic BM3 T cells (clonotype-positive, $\mathrm{CD}^{+}$) were sorted and tested for responsiveness to irradiated C57BL/6 spleen cells, with or without anti-CD3 antibody. In contrast, the unfractionated host $C D 8^{+}$population from the same LN showed good response to mitogen. (D) Response to third-party BALB/c antigens (irradiated BALB/c splenocytes, 5-day MLR) was intact in CBA+BM3 recipients primed with TDLN DCs, compared with normal CBA control mice.

$1-1.5 \%$ of cells; of these, the CD $19^{+}$pDCs comprised $31 \% \pm 15 \%$. Thus, the potent IDO-induced suppression seen in TDLNs was mediated by only $0.3-0.5 \%$ of total LN cells. The fact that CD $19^{+}$ pDCs mediated such potent suppression, whereas CD19+ B cells mediated none, effectively ruled out the possibility that the CD19+ pDCs were simply contaminating B cells.

$C D 19^{+}$DCs show a phenotype of pDCs. Figure 7A shows three-color staining for expression of B220 versus CD11c versus CD19 in a representative TDLN preparation. The $\mathrm{B} 220^{+} \mathrm{CD} 11 \mathrm{c}^{+}(\mathrm{pDC})$ cells were strongly bimodal with respect to CD19 expression, as shown in the upper dot plot. When the CD19+ and CD19- subsets of pDCs were gated separately, both were seen on forward scatter versus side scatter to be similar in properties to large lymphocytes (consistent with pDCs). In contrast, the B220-CD $11 \mathrm{c}^{+}$ (conventional) DCs were larger cells with greater side-scatter properties. Figure 7B shows immunophenotyping of the CD $11 \mathrm{c}^{+}$ cells from a TDLN preparation, gated separately on the CD19+ cells, versus all other CD19- DCs for comparison. Many of the CD19+ DCs expressed CD4 and/or CD8 $\alpha$, both of which can be found on $\mathrm{pDCs}$; this helped to further distinguish the CD19+

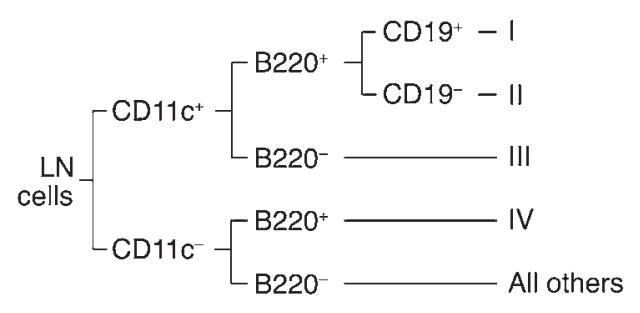

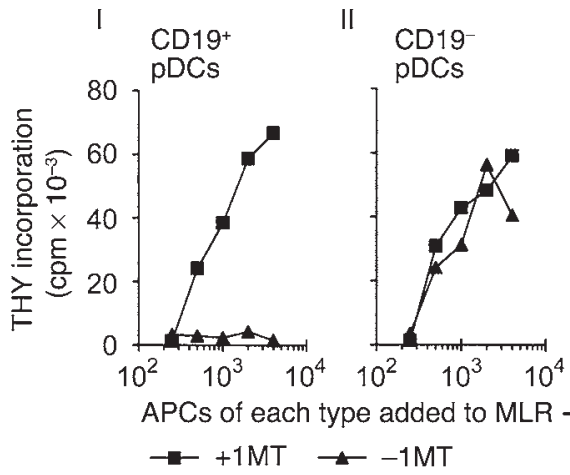

III

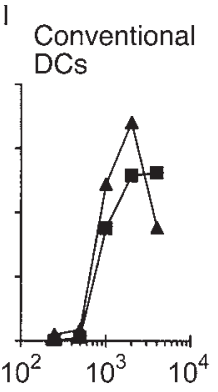

IV B cells (control)

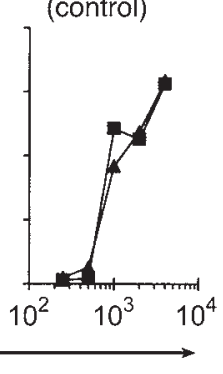

Figure 6

IDO-mediated suppressor activity segregates with a CD19+ subset of pDCs. Cells from TDLNs were sorted into five populations based on expression of CD11c, B220, and CD19, as shown in the schematic at the top. The three CD11c DC fractions (groups I, II, and III) or B cells (group IV) were used as stimulators in MLRs $\left(2 \times 10^{5} \mathrm{BM} 3\right.$ responder cells with a titration of stimulator cells), with and without $1 \mathrm{MT}$. Only the CD19+ (fraction I) showed significant IDO-mediated suppression. 
A
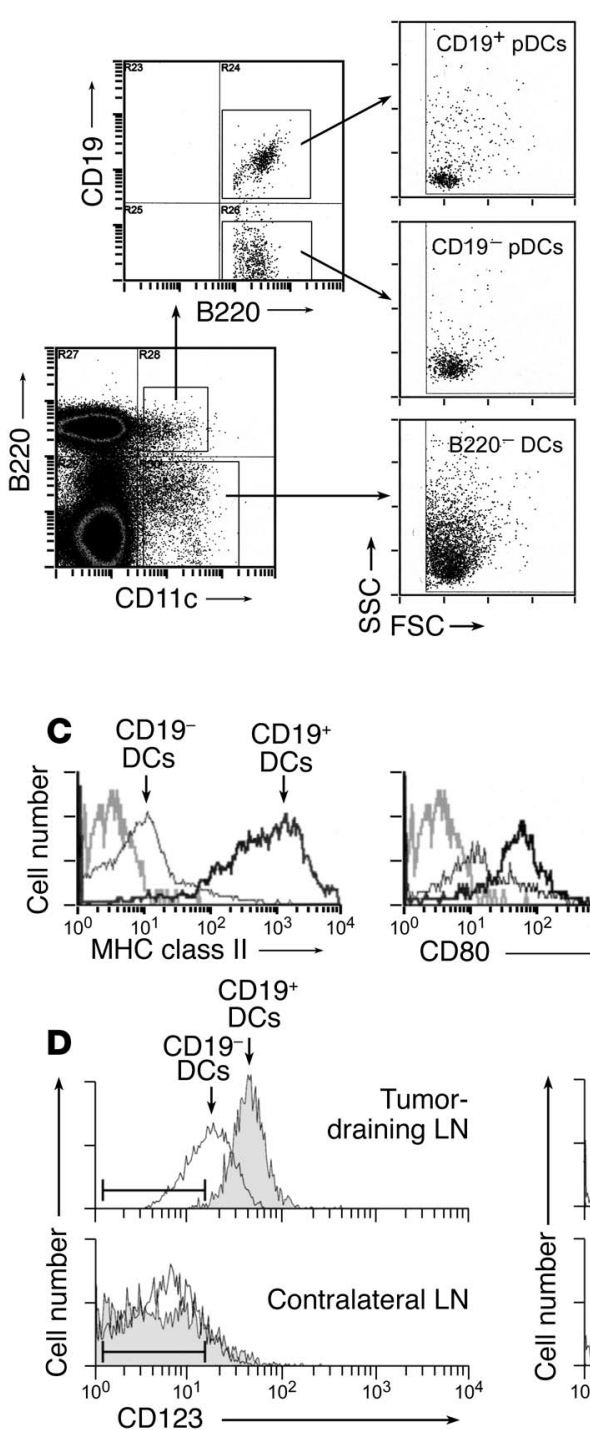

$\mathbf{B}$

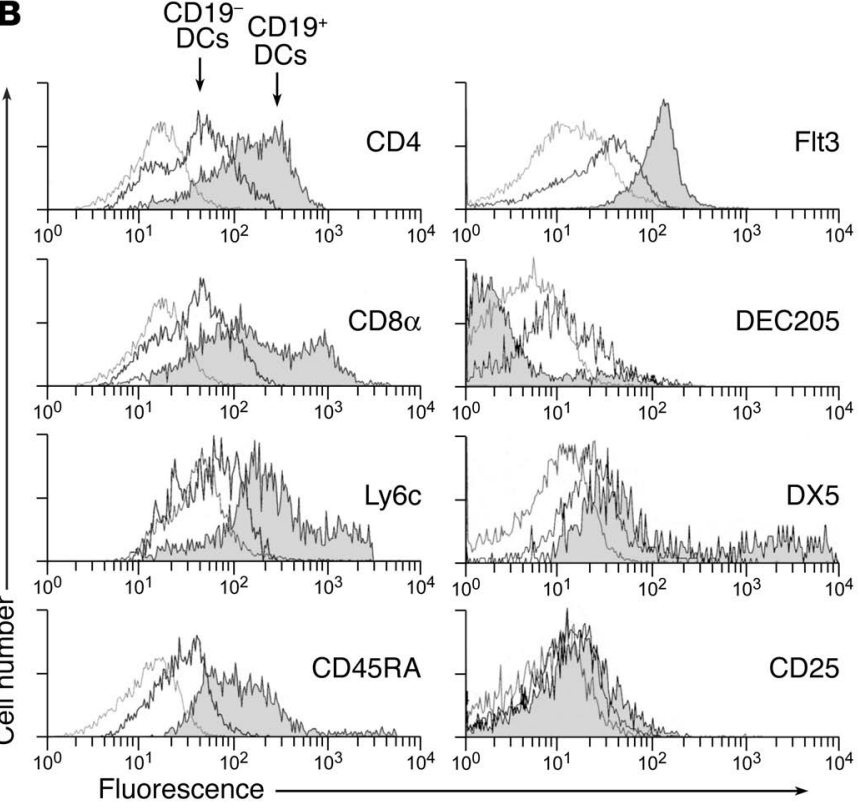

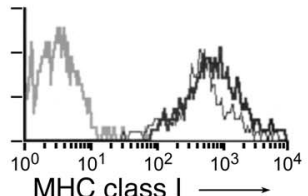
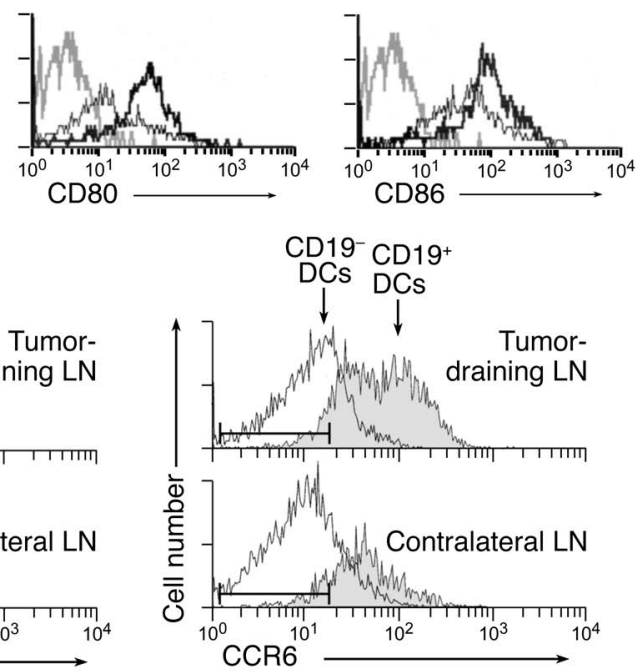

Figure 7

The CD19+ DC subset displays a phenotype consistent with pDCs. (A) Forward and side-scatter characteristics of B220- DCs versus B220+ DCs, with the latter further gated into CD19+ and CD19- subsets. (B) TDLNs stained by four-color flow cytometry for CD11C versus CD19 versus the various markers shown. Overlay histograms show the CD19+ (filled trace) and CD19- subsets of CD11 $\mathrm{C}^{+}$cells. Isotype-matched controls (gated on CD11 $\mathrm{c}^{+}$cells) are shown in gray. Each histogram is representative of 4-12 experiments with each marker. (C) TDLN DCs were stained for markers of DC maturity, and analyzed as above. (D) Expression of cell-surface markers CD123 and CCR6 on the CD19+ and CD19- DC subsets. Cells were analyzed from TDLNs and from contralateral LNs of the same animals, as shown. The range for the isotype-matched negative control antibody $( \pm 3 \mathrm{SD})$ is shown by the bars in each histogram.

DCs from B cells. Many of the CD19+ DCs also expressed the markers Ly6c and CD45RA, which are associated with murine pDCs $(40,41)$. The CD19+ DCs uniformly expressed the receptor tyrosine kinase Flt3 (CD135), which is not expressed on mature B cells (42). Expression of DEC205 was low to absent, and DX5 (expressed on the recently described tolerogenic NK-DCs [ref. 43]) was found on a subset of the CD19+ fraction. CD25 (which was included in some experiments as a marker of $\mathrm{T}$ cell activation) was negative on both the $\mathrm{CD} 19^{+}$and $\mathrm{CD} 19^{-}$fractions, as expected. Expression of CD19 mRNA in sorted CD19+ $\mathrm{pDCs}$ was confirmed by quantitative real-time PCR, as was expression of the obligate upstream transcription factor for CD19, pax5 (44, 45) (see Supplemental Figure 1; supplemental material available at http://www.jci.org/cgi/content/full/114/2/280/DC1).

Figure 7C shows that $\mathrm{CD} 19^{+}$pDCs from TDLNs uniformly expressed high levels of MHC class II, as well as costimulatory molecules CD80 and CD86, suggestive of a mature phenotype. These markers were expressed at levels equal to or greater than the levels expressed by conventional CD19- DCs from the same LN. (In TDLNs, the CD19- subset of DCs showed significant variability in the number of mature versus immature DCs; but in every experiment all of the $\mathrm{CD} 19^{+} \mathrm{pDCs}$ were invariably mature.) 
In other experiments (data not shown), $\mathrm{CD} 19^{+} \mathrm{pDCs}$ of the same phenotype $\left(\mathrm{CD} 11 \mathrm{c}^{+}, \mathrm{CD} 19^{+}, \mathrm{B} 220^{+}, \mathrm{Ly}_{6 \mathrm{c}^{+}}, \mathrm{CD} 45 \mathrm{RA}^{+}\right)$were also found in normal LNs from non-tumor-bearing hosts, as well as in the contralateral LNs of mice with B78H1.GM-CSF tumors. In the normal LNs they comprised $18 \% \pm 6 \%$ of total DCs, compared with $31 \% \pm 15 \%$ of total DCs in TDLNs of B78H1-GM-CSF tumors, and $19 \% \pm 2 \%$ of total DCs in TDLNs of B16F10 tumors. Thus, while there were was perhaps a modest increase in the number of CD19+ pDCs in TDLNs, they were also found in normal lymphoid tissue. The key difference was that $\mathrm{CD} 19^{+} \mathrm{pDCs}$ from normal tissues did not express functional IDO and were not constitutively suppressive.

In addition to expressing markers of murine $\mathrm{pDCs}$, the CD19+ pDCs in TDLNs also expressed two markers previously observed in human IDO $^{+}$APCs. We have reported that CD123 (IL-3R $\alpha$ chain) and the chemokine receptor CCR6 segregate closely with IDO expression in human monocyte-derived DCs and monocytederived macrophages $(20,46)$. In murine TDLNs, we found that expression of CD123 preferentially associated with the CD19+ subset of DCs in TDLNs (Figure 7D). In contrast, the majority of CD19- DCs were low or negative for these markers, as were all of the DCs (with or without CD19) from contralateral (non-tumordraining) LNs. A similar pattern was observed for CCR6, with preferential expression on the $\mathrm{CD} 19^{+}$subset, and higher expression in TDLNs compared with contralateral LNs.

\section{Discussion}

In the current study we identify a population of immunoregulatory DCs in TDLNs that are capable of mediating active immunosuppression in vitro, and of creating profound local T cell anergy in vivo. Previous studies of DCs isolated from tumors or TDLNs have observed a marked defect in their ability to stimulate T cells (47-50), which has usually been attributed to immaturity. However, it can be difficult to distinguish immaturity from more active forms of suppression (15). The key advance embodied in the current study is the application of specific experimental models (IDO inhibitors, IDO-KO mice, in vivo adoptive transfer) to unambiguously demonstrate the presence of active, dominant, IDO-mediated suppression in TDLNs.

An important attribute of IDO-mediated suppression was its fundamentally local nature. First, the $\mathrm{IDO}^{+}$cells themselves were localized, being found in TDLNs but not in other LNs from the same animal. Second, adoptive-transfer studies demonstrated that complete anergy was created in the local draining LNs, even though T cells at remote sites retained reactivity. From the perspective of the tumor, we hypothesize that local anergy may be all that is required. A number of studies have shown that tumors can be locally tolerated despite the systemic presence of competent, tumor-specific $\mathrm{T}$ cells $(5,11,51-54)$. Such tumors grow unchecked, demonstrating that immunosuppression does not have to be systemic in order to be effective. If all the LNs draining all the sites of tumor create local anergy, then the tumor will be de facto tolerated.

In this regard, it becomes relevant that IDO-mediated suppression was dominant over other, nonsuppressive APCs. This occurred even when antigen presentation was known to be restricted solely to IDO- APCs (compare Figure 3). Quantitatively, even a few percent of IDO-expressing pDCs was sufficient to suppress all T cell responses in vitro. Consistent with this, in our adoptive-transfer experiments the majority of transferred DCs were nonsuppressive (as shown by the fractionation experiments in Figure 6), yet all of the BM3 cells in the recipient LNs were rendered anergic. Anergy was antigen-specific (i.e., other host $\mathrm{T}$ cells remained responsive to mitogen, and to antigens not expressed on the transferred DCs). The antigen-specific nature of this anergy would not be incompatible with participation of a third-party (bystander) mode of suppression. As described in other systems $(55,56)$, induction of anergy may require both an antigen-nonspecific suppressive signal, and simultaneous signaling via the TCR (conferring antigen specificity). In our system, we postulate that anergy is induced in any $T$ cell that encounters antigen (regardless of the APC) in a milieu that is rendered immunosuppressive by IDO. Thus, we hypothesize that a small population of $\mathrm{IDO}^{+} \mathrm{pDCs}$ is able to redefine the entire TDLN as a tolerogenic milieu, even for tumor antigens being cross-presented by other, normally immunogenic APCs.

The downstream molecular mechanisms by which IDO is able to suppress neighboring $\mathrm{T}$ cells remains to be elucidated. The existence of some such mechanism has been inferred from previous reports showing that even a small minority of IDO-expressing DCs can dominantly suppress $\mathrm{T}$ cell responses in vivo (57-59). Conceptually, the suppressive effects of IDO fall into two categories: (a) those mediated by depletion of tryptophan, which include its antimicrobial $(60,61)$ and antiviral $(62,63)$ effects, and inhibition of $\mathrm{T}$ cell proliferation in some models $(18,25,64)$; and (b) effects mediated by toxic downstream metabolites of tryptophan, which include $\mathrm{CD}^{+} \mathrm{T}$ cell apoptosis $(65)$ and inhibition of $\mathrm{T}$ cell proliferation in other models $(66,67)$. In the case of third-party suppression, where inhibition must occur at a distance, a role for soluble factors such as tryptophan metabolites would seem intuitively likely, but experiments using the defined four-party system described in Figure 3 should help elucidate these mechanisms.

IDO-mediated suppressor activity in TDLNs segregated with a novel population of pDCs expressing CD19, a marker of the B cell lineage $(68,69)$. It is known that early $\mathrm{CD} 19^{+}$pro-B cells can give rise to DCs in vitro $(70,71)$, and recent analyses of both human and murine $\mathrm{pDCs}$ have suggested a B-lymphoid origin for a subset of pDCs $(72,73)$. Despite these observations, however, most previous studies have failed to appreciate the $\mathrm{CD} 19^{+}$subset of $\mathrm{pDCs}$; and where they have been described (74), they were excluded on the assumption that they were contaminating B cells. Previously, we have reported that pDCs constitute one of the "IDO-competent" subsets of DCs, able to upregulate IDO (by immunohistochemistry) in response to CTLA4-Ig treatment (58). In that earlier study we, like most investigators $(40,75)$, excluded $\mathrm{CD} 19^{+}$cells from our sorted pDC preparation (which we defined as $\left.\mathrm{CD} 11 \mathrm{c}^{+} \mathrm{B} 220^{+} \mathrm{CD} 19^{-}\right)$. We did not realize that - although the $\mathrm{CD} 19^{-} \mathrm{pDCs}$ did indeed express IDO by immunohistochemistry, as reported - we were excluding a functionally important subset of the pDCs. (In that study, suppressor activity was measured only in vivo, not on the sorted subsets.) In the current study, the development of micro-scale MLR assays allowed us to follow the functional IDO-mediated suppressor activity (not just protein expression), and hence we were able to identify the novel CD $19^{+} \mathrm{pDC}$ subset. It is not surprising that some subsets of DCs expressed IDO protein (by immunohistochemistry or Western blot) without significant suppressor activity, since this is well described in the literature (39). Thus, the conclusion of our earlier report, that $\mathrm{pDCs}$ are one of the IDO-competent DC subsets, remains unchanged; but we would now expand the definition of pDCs to include the functionally important CD $19^{+}$subset.

With the exception of their B-lineage attributes, the CD $19^{+} \mathrm{pDCs}$ expressed markers consistent with murine pDCs. Some DCs can display high autofluorescence and nonspecific binding, which may complicate analysis by FACS, so we were careful to ensure that 
our characterization of the $\mathrm{CD} 19^{+} \mathrm{pDCs}$ also rested on functional studies, not just on marker analysis. We show that expression of CD19 and pax5 mRNA segregated with surface CD19 staining (see supplemental material), and that the $\mathrm{CD} 19^{+}$pDCs contained essentially all of the IDO-mediated suppressor activity. Thus, by functional criteria, the $\mathrm{CD} 19^{+} \mathrm{pDCs}$ represented a distinct and important subset of DCs in TDLNs.

The $\mathrm{CD} 19^{+} \mathrm{pDCs}$ were not restricted to TDLNs. They were also found in normal LNs and spleen, but in these sites they did not constitutively express IDO and were not spontaneously suppressive. We have previously shown that administration of recombinant CTLA4-Ig in vivo upregulates IDO, preferentially affecting the $\mathrm{B} 220^{+}$and $\mathrm{CD} 8 \alpha^{+}$subsets of DCs (58). By analogy, we hypothesize that some factor in the TDLNs "pre-activates" the CD $19^{+} \mathrm{pDCs}$ for constitutive IDO-mediated suppression. This might be a microenvironmental signal (e.g., a local cytokine); or, as recently described (59), it might be a population of IDO-inducing Treg's. Elucidating these upstream inducing factors will be important in understanding how tumors have evolved to exploit the IDO mechanism.

The CD19+ subset of pDCs in TDLNs expressed CD123 and CCR6, which we have previously described as segregating preferentially with IDO-expressing human monocyte-derived DCs and monocyte-derived macrophages $(20,46)$. CCR6 has been described on at least a subset of murine pDCs (41), but (unlike the case in humans) CD123 has been reported to be negative on murine pDCs. However, CD123 expression may have been missed, since we found that there was essentially no CD123 expression on pDCs from normal (nontumor-draining) LNs (see Figure 7D). Even when pDCs were isolated from TDLNs, it was only the CD19+ $\mathrm{pDCs}$ that expressed significant levels of CD123 (and these cells are usually excluded from analysis). Thus, in mice as in humans, there may be a preferential association of CD123 and CCR6 as markers of IDO-expressing APCs. Whether the converse will prove true, that the human IDO ${ }^{+}$DCs in TDLNs resemble their murine $\mathrm{CD} 19^{+}$counterparts, remains to be determined. Others have shown that some human $\mathrm{pDCs}$ do indeed appear to arise from the B cell lineage (72), and we observe B cell markers such as CD20 on at least a subset of IDO-expressing cells in TDLNs (see Supplemental Figure 2). The key point, however, is that IDO-expressing human APCs (whatever the details of their immunophenotype) are found in human TDLNs, and they appear to correlate with poor clinical outcome in the patient population studied (Figure 1B).

The current study demonstrates that IDO-expressing DCs can be a potent endogenous immunosuppressive mechanism in tumorbearing mice. We know that IDO is not the only way that tumors evade the immune system, because IDO-KO mice are still susceptible to tumors, and tumors do not spontaneously regress in mice treated with $1 \mathrm{MT}$ alone (31). This is hardly surprising, however, since tumors deprived of any single protective mechanism will rapidly evolve escape variants (76). The salient point from a clinical perspective is that all human tumors will have arisen in IDO-sufficient hosts; therefore some tumors may have evolved to rely upon this mechanism for protection. The ability to acutely deprive these tumors of the protective IDO mechanism, by administering IDOinhibitor drugs such as $1 \mathrm{MT}$, may provide a therapeutic window in which to break tolerance to tumor antigens.

\section{Methods}

Clinical materials. Samples from 40 patients with malignant melanoma were randomly selected from the Moffitt Cancer Center archives, based on the following inclusion criteria: radiographically mapped sentinel LN at the time of diagnosis; no metastases to the sentinel node by light microscopy and immunohistochemistry; and no further therapy given following initial surgical resection. Sentinel LNs were stained for IDO by immunohistochemistry, expression was graded by two pathologists as previously described (30), and a consensus score was obtained. Patients were stratified into normal (grade 0 ) versus abnormal (grade 1+ or higher), and compared by Kaplan-Meier survival analysis. Sentinel LN biopsies from patients with breast cancer and melanoma were selected from the archives of the Medical College of Georgia. Studies using human subjects were approved by the respective Institutional Review Boards.

Mouse tumor models. All studies were performed under protocols approved by our institutional animal-use committee. Tumors were implanted in female C57BL/6 mice (The Jackson Laboratory, Bar Harbor, Maine, USA), 8-12 weeks of age, or in mice with a targeted disruption of the IDO gene (58). IDO-KO mice were self-mated $\mathrm{C} 57 \mathrm{BL} / 6 \times 129$ background, and C57BL/ 6 tumors grew in these mice; since both strains are $\mathrm{H} 2^{\mathrm{b}}$ haplotype, all mice were obligate homozygotes for the target $\mathrm{H} 2 \mathrm{~K}^{\mathrm{b}}$ antigen used in some experiments. Mice were implanted with tumors in the anteriomedial thigh, using either $4 \times 10^{4}$ B16F10 cells (American Type Culture Collection, Bethesda, Maryland, USA) or $1 \times 10^{6} \mathrm{~B} 78 \mathrm{H} 1 \cdot \mathrm{GM}-\mathrm{CSF}$ cells (32) (gift of H. Levitsky). The B78H1-GM-CSF cells were originally designed as vaccine adjuvants (77), since they recruit large numbers of APCs to the tumor and TDLN (33). However, while GM-CSFtransfected tumor cells are immunogenic if irradiated prior to injection, live GM-CSF-expressing tumors paradoxically create systemic tolerance to tumor antigens (78). Thus, B78H1.GM-CSF offered an excellent model for our studies, because it recruited many DCs (including $\mathrm{IDO}^{+} \mathrm{DCs}$ ) yet failed to provoke a protective immune response. Although this model was intentionally biased toward recruitment of IDO-expressing cells, it was a biologically relevant model system, since similar cells were found in nontransfected tumors (B16F10 and LLC), and many human tumors and TDLNs spontaneously express GM-CSF (78-80). FACS analysis showed that the pDCs recruited by B78H1.GM-CSF tumors were phenotypically identical to those recruited by B16F10 tumors but could be recovered in larger quantities. Tumors were used 10-12 days after implantation (5-7 mm diameter), well prior to any metastasis to spleen or draining LNs (which occurred at day 20-30).

In some experiments, mice received 1MT (NSC 721782; National Cancer Institute, Rockville, Maryland, USA) as a continuous subcutaneous infusion, using implantable copolymer pellets as previously described (21). Control mice received vehicle pellets alone.

Immunohistochemistry. Immunohistochemistry was performed on human materials as previously described (20). Formalin-fixed paraffin-embedded mouse tissues were treated with proteinase $K$ antigen retrieval prior to staining with rabbit anti-mouse IDO polyclonal antibody (58). The IDO epitope is not stable under conditions of prolonged storage in thin sections, so staining was performed within 24 hours of sectioning. Negative controls for human and mouse staining were the anti-IDO antibody neutralized with a 50-fold molar excess of the immunizing peptide. Multiple immunohistochemical studies of established B16F10 and B78H1-GM-CSF tumors showed that neither tumor expressed IDO in the tumor cells themselves. Consistent with this, the studies presented herein using IDO-deficient hosts formally showed that the relevant IDO expression was in the host-derived APCs, not in the tumor cell lines.

Flow cytometry and cell sorting. Single-cell suspensions of LNs were obtained by teasing and disaggregation through a $40-\mu \mathrm{m}$ mesh; 
spleen cells were obtained by ground-glass homogenization and hypotonic lysis of erythrocytes. Cells were stained by four-color immunofluorescence, using CD11c versus B220 versus CD19 versus a panel of other markers. Fc binding was blocked using a commercial anti-CD16/CD32 cocktail (BD Biosciences - Pharmingen, San Diego, California, USA). Acquisition and sorting were performed using pulse-processing doublet discrimination on a MoFlo cell sorter (Cytomation Inc., Ft. Collins, Colorado, USA). (Analysis was also performed on a four-color FACSCalibur [BD Biosciences - Immunocytometry Systems, San Jose, California, USA] with similar results.) Antibodies against the following antigens were from BD Biosciences - Pharmingen: CD11c (clone HL3), B220 (clone RA3-6B2), CD19 (clone 1D3), CD4 (clone H129.19), CD8 $\alpha$ (clone 53.6.7), Ly6c (clone AL-21), CD45RA (clone HI100), MHC class II (anti-H2A b clone 25-9-17), CD80 (clone 16-10A1), CD86 (clone GL1), H2 K ${ }^{\mathrm{b}}$ (clone AF6-88.5), CD123 (clone 5B11), CD135/Flt3 (clone A2F10.1), CD49b/DX5 (clone DX5), CD25 (clone PC61). DEC205-FITC (clone NLDC-145) was obtained from Serotec Inc. (Raleigh, North Carolina, USA). Anti-mouse CCR6 (clone 140706) was from R\&D Systems Inc. (Minneapolis, Minnesota, USA). Anticlonotypic antibody Ti98 against the BM3 TCR was biotinylated and used as previously described (34). All antibodies were used with isotype-matched negative controls; each isotype control was gated on the specific DC population of interest.

$T$ cell activation in MLR. TCR-transgenic BM3 responder $\mathrm{T}$ cells (CBA background, anti-H2 $\mathrm{K}^{\mathrm{b}}$ [ref. 34]) were prepared from spleen by nylon-wool enrichment. Stimulator cells (either sorted DCs or unfractionated TDLN cells) were mixed with $1 \times 10^{5} \mathrm{BM} 3$ responder cells at the ratios shown in each figure, and cultured in $200 \mu \mathrm{l}$ medium (10\% FCS in RPMI-1640, with antibiotics and $50 \mu \mathrm{M} 2$-mercaptoethanol). Anti-HY TCR-transgenic mice (CD4 ${ }^{+}$, clone A1, CBA background) have been described previously (81). These $T$ cells recognize the cognate peptide REEALHQFRSGRKPI in the context of $\mathrm{H} 2 \mathrm{E}^{\mathrm{k}}(82)$. CD11 $\mathrm{c}^{+} \mathrm{DCs}$ were sorted from spleens of normal female CBA mice, pulsed for 90 minutes with $100 \mu \mathrm{M}$ cognate peptide, washed thoroughly, and used as APCs in MLRs.

All MLR assays were performed in quadruplicate. After 3 days, proliferation was measured by 4-hour thymidine incorporation assay. All MLRs were performed in V-bottom culture wells (Nalge Nunc International, Rochester, New York, USA), as previously described (20), in order to ensure the close cell-cell contact required for optimal sensitivity to IDO-mediated suppression. Stimulator DCs were not irradiated, because preliminary validation studies showed that irradiation significantly altered the viability and functional attributes of $\mathrm{IDO}^{+} \mathrm{pDCs}$. MLRs were thus "two-way" reactions; however, the small number of sorted DCs used as stimulators contributed negligible proliferation compared with the large population of TCR-transgenic responder cells. Since the relevant readout was dominant suppression of all T cell proliferation, the two-way MLR design presented no problem in interpretation. Where indicated, replicate groups of wells received $200 \mu \mathrm{M} 1 \mathrm{MT}$ (Sigma-Aldrich) or $250 \mu \mathrm{M}$ L-tryptophan (Sigma-Aldrich). (Stock solution of $20 \mathrm{mM} 1 \mathrm{MT}$ was dissolved in $0.1 \mathrm{~N} \mathrm{NaOH}$, then adjusted to $\mathrm{pH}$ 7.4.)

Adoptive-transfer studies. Recipient CBA mice (The Jackson Laboratory) were prepared by intravenous injection of $4 \times 10^{7}$ congenic $\mathrm{BM} 3$ splenocytes, to generate "CBA+BM3" hosts. Adoptively transferred BM3 T cells are stable in CBA hosts for over 100 days (34). CD $11 c^{+}$DCs were isolated from TDLNs, or from normal LNs of non-tumor-bearing C57BL/6 and CBA mice, using a Cytomation Inc. MoFlo high-speed cell sorter. Two aliquots of $5 \times 10^{4} \mathrm{DCs}$ each were injected subcutaneously into each recipient, bilaterally into the anteriomedial thigh (analogous to the position of the original tumor). After 10-12 days, recipient mice were euthanized, and the inguinal LNs (draining the sites of injection) were removed for analysis. Spleens were also harvested, as a representative distant site.

\section{Acknowledgments}

We thank Joyce Wilson and Jingping Sun for expert technical assistance, Jeanene Pihkala and the Medical College of Georgia Flow Cytometry Core Facility for cell sorting, and Alan Cantor for statistical analysis. This work was supported by NIH grants R01 CA103220 and R01 CA096651 (to D.H. Munn), R01 HD41187 (to A.L. Mellor), and the Carlos and Marguerite Mason Trust.

Received for publication March 15, 2004, and accepted in revised form May 25, 2004.

Address correspondence to: David H. Munn, Institute for Molecular Medicine and Genetics, Room CA-2010, Medical College of Georgia, Augusta, Georgia 30912, USA. Phone: (706) 721-7141; Fax: (706) 721-8732; E-mail: dmunn@mail.mcg.edu.
1. Smyth, M.J., Godfrey, D.I., and Trapani, J.A. 2001. A fresh look at tumor immunosurveillance and immunotherapy. Nat. Immunol. 2:293-299.

2. Staveley-O'Carroll, K., et al. 1998. Induction of antigen-specific $\mathrm{T}$ cell anergy: an early event in the course of tumor progression. Proc. Natl. Acad. Sci. U. S. A. 95:1178-1183.

3. Ochsenbein, A.F., et al. 2001. Roles of tumour localization, second signals and cross priming in cytotoxic T-cell induction. Nature. 411:1058-1064.

4. Spiotto, M.T., et al. 2002. Increasing tumor antigen expression overcomes "ignorance" to solid tumors via crosspresentation by bone marrow-derived stromal cells. Immunity. 17:737-747.

5. Wick, M., et al. 1997. Antigenic cancer cells grow progressively in immune hosts without evidence for T cell exhaustion or systemic anergy. J. Exp. Med. 186:229-238.

6. Gallimore, A., and Sakaguchi, S. 2002. Regulation of tumour immunity by CD25+ T cells. Immunology. 107:5-9.

7. Pardoll, D. 2001. T cells and tumours. Nature. 411:1010-1012.

8. Sotomayor, E.M., et al. 2001. Cross-presentation of tumor antigens by bone marrow-derived antigenpresenting cells is the dominant mechanism in the induction of T-cell tolerance during B-cell lymphoma progression. Blood. 98:1070-1077.

9. Cuenca, A., et al. 2003. Extra-lymphatic solid tumor growth is not immunologically ignored and results in early induction of antigen-specific T-cell anergy: dominant role of cross-tolerance to tumor antigens. Cancer Res. 63:9007-9015.

10. Yu, P., Spiotto, M.T., Lee, Y., Schreiber, H., and Fu, Y.X. 2003. Complementary role of CD4+ T cells and secondary lymphoid tissues for cross-presentation of tumor antigen to CD8+ T cells. J. Exp. Med. 197:985-995.

11. Nguyen, L.T., et al. 2002. Tumor growth enhances cross-presentation leading to limited $\mathrm{T}$ cell activation without tolerance. J. Exp. Med. 195:423-435.

12. Spiotto, M.T., Fu, Y.X., and Schreiber, H. 2003. Tumor immunity meets autoimmunity: antigen levels and dendritic cell maturation. Curr. Opin. Immunol. 15:725-730.

13. Melief, C.J. 2003. Mini-review. Regulation of cytotoxic $\mathrm{T}$ lymphocyte responses by dendritic cells: peaceful coexistence of cross-priming and direct priming? Eur. J. Immunol. 33:2645-2654.

14. Steinman, R.M., Hawiger, D., and Nussenzweig, M.C. 2003. Tolerogenic dendritic cells. Annu. Rev. Immunol. 21:685-711.

15. Moser, M. 2003. Dendritic cells in immunity and tolerance: do they display opposite functions? Immunity. 19:5-8.

16. Mellor, A.L., and Munn, D.H. 2003. Tryptophan catabolism and regulation of adaptive immunity. J. Immunol. 170:5809-5813.

17. Grohmann, U., Fallarino, F., and Puccetti, P. 2003. Tolerance, DCs and tryptophan: much ado about IDO. Trends Immunol. 24:242-248.

18. Munn, D.H., et al. 1999. Inhibition of T cell proliferation by macrophage tryptophan catabolism. J. Exp. Med. 189:1363-1372.

19. Hwu, P., et al. 2000. Indoleamine 2,3-dioxygenase production by human dendritic cells results in the inhibition of $\mathrm{T}$ cell proliferation. J. Immunol. 164:3596-3599.

20. Munn, D.H., et al. 2002. Potential regulatory function of human dendritic cells expressing indoleamine 2,3-dioxygenase. Science. 297:1867-1870.

21. Munn, D.H., et al. 1998. Prevention of allogeneic 
fetal rejection by tryptophan catabolism. Science. 281:1191-1193.

22. Grohmann, U., et al. 2003. A defect in tryptophan catabolism impairs tolerance in nonobese diabetic mice. J. Exp. Med. 198:153-160.

23. Grohmann, U., et al. 2002. CTLA-4-Ig regulates tryptophan catabolism in vivo. Nat. Immunol. 3:985-1109.

24. Gurtner, G.J., Newberry, R.D., Schloemann, S.R., McDonald, K.G., and Stenson, W.F. 2003. Inhibition of indoleamine 2,3-dioxygenase augments trinitrobenzene sulfonic acid colitis in mice. Gastroenterology. 125:1762-1773.

25. Sakurai, K., Zou, J., Tschetter, J., Ward, J., and Shearer, G. 2002. Effect of indoleamine 2,3-dioxygenase on induction of experimental autoimmune encephalomyelitis. J. Neuroimmunol. 129:186-196.

26. Munn, D.H., and Mellor, A.L. 2004. IDO and tolerance to tumors. Trends Mol. Med. 10:15-18.

27. Uyttenhove, C., et al. 2003. Evidence for a tumoral immune resistance mechanism based on tryptophan degradation by indoleamine 2,3-dioxygenase. Nat.Med. 9:1269-1274.

28. Taylor, M.W., and Feng, G. 1991. Relationship between interferon- $\gamma$, indoleamine 2,3-dioxygenase, and tryptophan catabolism. FASEB J. 5:2516-2522.

29. Mellor, A.L., Keskin, D.B., Johnson, T., Chandler, P., and Munn, D.H. 2002. Cells expressing indoleamine 2,3 dioxygenase inhibit $\mathrm{T}$ cell responses. J. Immunol. 168:3771-3776.

30. Lee, J.R., et al. 2003. Pattern of recruitment of immunoregulatory antigen presenting cells in malignant melanoma. Lab. Invest. 83:1457-1466.

31. Friberg, M., et al. 2002. Indoleamine 2,3-dioxygenase contributes to tumor cell evasion of T cell-mediated rejection. Int. J. Cancer. 101:151-155.

32. Huang, A.Y., et al. 1994. Role of bone marrowderived cells in presenting MHC class I-restricted tumor antigens. Science. 264:961-965.

33. Dranoff, G., et al. 1993. Vaccination with irradiated tumor cells engineered to secrete murine granulocyte-macrophage colony-stimulating factor stimulates potent, specific, and long-lasting anti-tumor immunity. Proc. Natl. Acad. Sci. U. S. A. 90:3539-3543

34. Tarazona, R., et al. 1996. Effects of different antigenic microenvironments on the course of $\mathrm{CD}^{+} \mathrm{T}$ cell responses in vivo. Int. Immunol. 8:351-358.

35. Sutmuller, R.P.M., et al. 2001. Synergism of cytotoxic $\mathrm{T}$ lymphocyte-associated antigen 4 blockade and depletion of CD25+ regulatory T cells in antitumor therapy reveals alternative pathways for suppression of autoractive cytotoxic T lymphocyte responses. J. Exp. Med. 194:823-832.

36. Hogquist, K.A., et al. 1994. T cell receptor antagonist peptides induce positive selection. Cell. 76:17-27.

37. Schwartz, R.H. 1996. Models of T cell anergy: is there a common molecular mechanism? J. Exp. Med. 184:1-8.

38. Shrikant, P., Khoruts, A., and Mescher, M.F. 1999. CTLA-4 blockade reverses CD8+ T cell tolerance to tumor by a CD4+ T cell- and IL-2-dependent mechanism. Immunity. 11:483-493.

39. Fallarino, F., et al. 2002. Functional expression of indoleamine 2,3-dioxygenase by murine CD8alpha $(+)$ dendritic cells. Int. Immunol. 14:65-68.

40. O'Keeffe, M., et al. 2002. Mouse plasmacytoid cells: long-lived cells, heterogeneous in surface phenotype and function, that differentiate into CD8(+) dendritic cells only after microbial stimulus. J. Exp. Med. 196:1307-1319.

41. Martin, P., et al. 2002. Characterization of a new subpopulation of mouse CD8alpha+ B220+ dendritic cells endowed with type 1 interferon production capacity and tolerogenic potential. Blood. 100:383-390.

42. Karsunky, H., Merad, M., Cozzio, A., Weissman, I.L., and Manz, M.G. 2003. Flt3 ligand regulates dendritic cell development from Flt3+ lymphoid and myeloid-committed progenitors to Flt $3+$ dendritic cells in vivo. J. Exp. Med. 198:305-313.

43. Homann, D., et al. 2002. CD40L blockade prevents autoimmune diabetes by induction of bitypic NK/ DC regulatory cells. Immunity. 16:403-415.

44. Nutt, S.L., Morrison, A.M., Dorfler, P., Rolink, A., and Busslinger, M. 1998. Identification of BSAP $(\mathrm{Pax}-5)$ target genes in early B-cell development by loss- and gain-of-function experiments. EMBO J. 17:2319-2333.

45. Mikkola, I., Heavey, B., Horcher, M., and Busslinger, M. 2002. Reversion of B cell commitment upon loss of Pax5 expression. Science. 297:110-113.

46. Munn, D.H., Sharma, M.D., and Mellor, A.L. 2004 Ligation of B7-1/B7-2 by human CD4+ T cells triggers indoleamine 2,3-dioxygenase activity in dendritic cells. J. Immunol. 172:4100-4110.

47. Vicari, A.P., et al. 2002. Reversal of tumor-induced dendritic cell paralysis by $\mathrm{CpG}$ immunostimulatory oligonucleotide and anti-interleukin 10 receptor antibody. J. Exp. Med. 196:541-549.

48. Almand, B., et al. 2000. Clinical significance of defective dendritic cell differentiation in cancer. Clin. Cancer Res. 6:1755-1766.

49. Yang, L., et al. 2003. Cancer-associated immunodeficiency and dendritic cell abnormalities mediated by the prostaglandin EP2 receptor. J. Clin. Invest. 111:727-735. doi:10.1172/JCI200316492.

50. Furumoto, K., Soares, L., Engleman, E.G., and Merad, M. 2004. Induction of potent antitumor immunity by in situ targeting of intratumoral DCs. J. Clin. Invest. 113:774-783. doi:10.1172/ JCI200419762.

51. Hanson, H.L., et al. 2000. Eradication of established tumors by CD8 $+\mathrm{T}$ cell adoptive immunotherapy. Immunity. 13:265-276.

52. Nelson, D.J., et al. 2001. Tumor progression despite efficient tumor antigen cross-presentation and effective "arming" of tumor antigen-specific CTL. J. Immunol. 166:5557-5566.

53. Speiser, D.E., et al. 1997. Self antigens expressed by solid tumors do not efficiently stimulate naive or activated T cells: implications for immunotherapy. J. Exp. Med. 186:645-653.

54. Overwijk, W.W., et al. 2003. Tumor regression and autoimmunity after reversal of a functionally tolerant state of self-reactive CD8+ T cells. J. Exp. Med. 198:569-580

55. DeSilva, D.R., Urdahl, K.B., and Jenkins, M.K 1991. Clonal anergy is induced in vitro by $\mathrm{T}$ cell receptor occupancy in the absence of proliferation. J. Immunol. 147:3261-3267.

56. Powell, J.D., Lerner, C.G., and Schwartz, R.H. 1999. Inhibition of cell cycle progression by rapamycin induces $\mathrm{T}$ cell clonal anergy even in the presence of costimulation. J. Immunol. 162:2775-2784.

57. Grohmann, U., et al. 2000. IFN-gamma inhibits presentation of a tumor/self peptide by CD8 alphadendritic cells via potentiation of the CD8 alpha+ subset. J. Immunol. 165:1357-1363.

58. Mellor, A.L., et al. 2003. Cutting edge: induced indoleamine 2,3 dioxygenase expression in dendritic cell subsets suppresses $\mathrm{T}$ cell clonal expansion. J. Immunol. 171:1652-1655.

59. Fallarino, F., et al. 2003. Modulation of tryptophan catabolism by regulatory $\mathrm{T}$ cells. Nat. Immunol. 4:1206-1212.

60. Pfefferkorn, E.R. 1984. Interferon $\gamma$ blocks the growth of Toxoplasma gondii in human fibroblasts by inducing the host cells to degrade tryptophan. Proc. Natl. Acad. Sci. U. S. A. 81:908-912.

61. Gupta, S.L., et al. 1994. Antiparasitic and antiproliferative effects of indoleamine 2,3-dioxygenase enzyme expression in human fibroblasts. Infect. Immun. 62:2277-2284.

62. Adams, O., et al. 2004. Role of indoleamine-2,3 dioxygenase in alpha/beta and gamma interferon- mediated antiviral effects against herpes simplex virus infections. J. Virol. 78:2632-2636.

63. Bodaghi, B., et al. 1999. Role of IFN-gammainduced indoleamine 2,3 dioxygenase and inducible nitric oxide synthase in the replication of human cytomegalovirus in retinal pigment epithelial cells. J. Immunol. 162:957-964.

64. Bubnoff, D., et al. 2002. Fc-gamma RI induces the tryptophan degradation pathway involved in regulating T cell responses. J. Immunol. 169:1810-1816.

65 . Fallarino, F., et al. 2002. T cell apoptosis by tryptophan catabolism. Cell Death Differ. 9:1069-1077.

66. Frumento, G., et al. 2002. Tryptophan-derived catabolites are responsible for inhibition of $\mathrm{T}$ and natural killer cell proliferation induced by indoleamine 2,3-dioxygenase. J. Exp. Med. 196:459-468

67. Terness, P., et al. 2002. Inhibition of allogeneic T cell proliferation by indoleamine 2,3-dioxygenase-expressing dendritic cells: mediation of suppression by tryptophan metabolites. J. Exp. Med. 196:447-457

68. Fearon, D.T., and Carroll, M.C. 2000. Regulation of $B$ lymphocyte responses to foreign and self-antigens by the CD19/CD21 complex. Annu. Rev. Immunol. 18:393-422.

69. Nutt, S.L., Heavey, B., Rolink, A.G., and Busslinger, M. 1999. Commitment to the B-lymphoid lineage depends on the transcription factor Pax5. Nature. 401:556-562.

70. Bjorck, P., and Kincade, P.W. 1998. CD19+ pro$B$ cells can give rise to dendritic cells in vitro. J. Immunol. 161:5795-5799.

71. Izon, D., et al. 2001. A common pathway for dendritic cell and early B cell development. J. Immunol. 167:1387-1392.

72. Rissoan, M.C., et al. 2002. Subtractive hybridization reveals the expression of immunoglobulin-like transcript 7, Eph-B1, granzyme B, and 3 novel transcripts in human plasmacytoid dendritic cells. Blood. 100:3295-3303

73. Corcoran, L., et al. 2003. The lymphoid past of mouse plasmacytoid cells and thymic dendritic cells. J. Immunol. 170:4926-4932.

74. Iwasaki, A., and Kelsall, B.L. 1999. Freshly isolated Peyer's patch, but not spleen, dendritic cells produce interleukin 10 and induce the differentiation of $\mathrm{T}$ helper type 2 cells. J. Exp. Med. 190:229-239.

75. Asselin-Paturel, C., et al. 2001. Mouse type I IFNproducing cells are immature APCs with plasmacytoid morphology. Nat. Immunol. 2:1144-1150.

76. Khong, H.T., and Restifo, N.P. 2002. Natural selection of tumor variants in the generation of "tumor escape" phenotypes. Nat. Immunol. 3:999-1005.

77. Borrello, I., Sotomayor, E.M., Cooke, S., and Levitsky, H.I. 1999. A universal granulocyte-macrophage colony-stimulating factor-producing bystander cell line for use in the formulation of autologous tumor cell-based vaccines. Hum. Gene Ther. 10:1983-1991.

78. Bronte, V., et al. 1999. Unopposed production of granulocyte-macrophage colony-stimulating factor by tumors inhibits CD8+ T cell responses by dysregulating antigen-presenting cell maturation. J. Immunol. 162:5728-5737.

79. Mattei, S., et al. 1994. Expression of cytokine/growth factors and their receptors in human melanoma and melanocytes. Int. J. Cancer. 56:853-857.

80. Leong, S.P., Peng, M., Zhou, Y.M., Vaquerano, J.E., and Chang, J.W. 2002. Cytokine profiles of sentinel lymph nodes draining the primary melanoma. Ann. Surg. Oncol. 9:82-87.

81. Zelenika, D., et al. 1998. Rejection of H-Y disparate skin grafts by monospecific CD4+ Th1 and Th2 cells: no requirement for CD8+ $\mathrm{T}$ cells or B cells. J. Immunol. 161:1868-1874.

82. Scott, D., et al. 2000. Dendritic cells permit identification of genes encoding MHC class II-restricted epitopes of transplantation antigens. Immunity. 12:711-720. 